\section{Hydrogen therapy may be a promising, safe and effective treatment for diabetic erectile dysfunction: a hypothesis}

\author{
Jun Chen, 1 Bin Zhang,1 Mingchao Li, 2 \\ Tao Qi, ${ }^{1}$ Zheng Chen, ${ }^{1}$ Xuejun Sun, ${ }^{3}$ \\ Xiao Chen ${ }^{3}$ \\ 1Department of Infertility and Sexual \\ Medicine, The Third Affiliated Hospital, \\ Sun Yat-Sen University, Guangzhou; \\ 2Department of Urology, Tongji Hospital, \\ Tongji Medical College, Huazhong \\ University of Science and Technology, \\ Wuhan; 3Department of Diving Medicine, \\ Second Military Medical University, \\ Shanghai, PR China
}

\section{Abstract}

Inhalation of hydrogen gas has been proven to be an effective treatment for ischemia-reperfusion injury by selectively reducing hydroxyl and peroxynitrite radicals. There has been considerable evidence of hydrogen's protective effect against diseases related to oxidative injury, such as the ischemia-reperfusion injury of the brain, liver and heart. More and more studies suggest that radical oxygen species (ROS) play an important role in the development of diabetic erectile dysfunction (ED) and antioxidants can markedly decrease the production of ROS and improve the erectile function. We hypothesize that hydrogen therapy may be a promising, safe and effective treatment for diabetic ED by reducing the production of ROS.

\section{Introduction}

Hydrogen is the lightest and most essential chemical element, composing nearly $75 \%$ of the universe's elemental matter. ${ }^{1}$ Hydrogen is seldom regarded as an important candidate in medicine, especially as a therapeutic gas. ${ }^{2}$ However, a recent study showed that hydrogen inhalation exhibited antioxidant and antiapoptotic activities which protected the brain against ischemia-reperfusion injury by selectively reducing hydroxyl radical and peroxynitrite, which are much more reactive than other ROS. ${ }^{3}$ Other researchers have indicated that hydrogen can also improve myocardial, hepatic ischemia-reperfusion injury, neonatal hypoxia-ischemia, Parkinson's disease; oxidative stress induced cognitive decline and diabetes mellitus (DM). ${ }^{4-9}$

Oxidative stress-mediated neurovascular alteration appears to play an integral role in the development of erectile dysfunction (ED) in diabetic populations. ${ }^{10,11} \mathrm{~N} 0$, the principal mediator of penile erection can react with other radicals, such as superoxide anions, to produce peroxynitrite and contribute to numerous pathological conditions such as atherosclerosis and generalized reperfusion injury. ${ }^{12,13}$ Hydroxyl radical is generated in biological systems from superoxide anions and hydrogen peroxide by the Haber-Weiss reaction or from hydrogen peroxide by the Fenton reaction, ${ }^{14,15}$ which is the strongest of the oxidant species and reacts indiscriminately with nucleic acids, lipids and proteins. ${ }^{3}$ A growing number of studies suggested that radical oxygen species (ROS) play an important role in the development of diabetic ED and antioxidants can markedly decrease the production of ROS and improve the erectile function. However, no study has been conducted to investigate the effects of hydrogen in the treatment of diabetic $\mathrm{ED}$, in which ROS play a pivotal role.

\section{Hydrogen is an important} physiological regulatory factor with antioxidant protective effects which selectively reduces hydroxyl radical and peroxynitrite generation

Hydrogen is the lightest and most abundant of chemical elements, constituting nearly $75 \%$ of the universe's elemental mass. ${ }^{1}$ It is a colorless, odorless, nonmetallic, tasteless, highly flammable diatomic gas which is mainly used in fossil fuel processing and ammonia production. ${ }^{16}$ Recent basic and clinical research has revealed that hydrogen is an important physiological regulatory factor with antioxidant protective effects on cells and organs. ${ }^{17}$

For both stroke and myocardial infarction there are now highly successful approaches to restore blood flow to the ischemic tissue. So far, however, research has failed to relieve this pathological cascade of oxidative damage after reperfusion injury, the burst of reactive oxygen species irrevocably drives downstream signaling networks that lead to cellular necrosis and apoptosis. In this issue, Ohsawa et al. report that highly diffusible hydrogen gas can target intracellular sources of reactive oxygen species and dose-dependently inhibit reperfusion-induced oxidative damage. $\mathrm{H} 2$ selectively reduces hydroxyl radical and peroxynitrite in vitro and in vivo, which are much more reactive than other ROS. ${ }^{3}$

A recent study demonstrated that inhalation of $\mathrm{H} 2$, at an incombustible level, limited the extent of myocardial infarction resulting from myocardial ischemia-reperfusion injury, and thereby preserved left ventricular (LV) function in vivo and ex vivo. The anti-oxidant action of molecular $\mathrm{H} 2$ may be explained, at least partially, by direct ROS scavenging
Correspondence: Jun Chen, Department of Infertility and Sexual Medicine, the Third Affiliated Hospital of Sun Yat-Sen University, Guangzhou, Guangdong 510630, China.

Tel: +86.20.85253235 - Fax: +86.20.87334510.

E-mail: jchen121121@hotmail.com

Bin Zhang, Department of Infertility and Sexual Medicine, the Third Affiliated Hospital of Sun Yat-Sen University, Guangzhou, Guangdong 510630, China.

Tel: +86.20 .85253235 - Fax: +86.20 .87334510 . E-mail: doc305@163.com.

Key words: gas medicine, hydrogen therapy, diabetic erectile dysfunction.

Acknowledgments: this study was supported by National Natural Sciences Foundation of China (No. 81070487), Doctoral Fund of Ministry of Education of China (No.20090171120078), Science and Technology Planning Project of Guangdong Province, China (2010B031600038), Medical Scientific Research Foundation of Guangdong Province, China (No. A2009190), the Fundamental Research Funds for theCentral Universities, Asia Pacific Society for Sexual Medicine Research Grant (No.2008-2), and Pfizer Asia Urology Research Grant (WS570255 ).

Conflict of interest: the authors report no conflicts of interest.

Received for publication: 4 July 2011. Accepted for publication: 30 August 2011.

This work is licensed under a Creative Commons Attribution NonCommercial 3.0 License (CC BYNC 3.0).

(C) Copyright J. Chen et al., 2011

Licensee PAGEPress, Italy

Alternative Medicine Studies 2011; 1:e11

doi:10.4081/ams.2011.e11

effect. ${ }^{4}$ Hydrogen-rich saline improved postischemic functional recovery of rat hearts. The improvement in post-ischemic functional recovery was paralleled by a significant reduction in infarct size, decreased plasma and myocardium malondialdehyde (MDA) concentration, attenuation of cardiac cell apoptosis and DNA oxidative stress in at risk areas (AAR). Histological analysis revealed a substantial decrease in hemorrhage and necrosis as well as a decrease in the number of leukocytes within the ischemic zone. This cardiac improvement may result from radical oxygen species (ROS) scavenging effect of molecular H2. 5 The inhalation of hydrogen gas was applicable for hepatic injury caused by ischemia/reperfusion using mice. The portal triad to the left lobe and the left middle lobe of the liver were completely occluded for $90 \mathrm{~min}$, followed by reperfusion for $180 \mathrm{~min}$. Inhalation of hydrogen gas (1-4\%) during the last $190 \mathrm{~min}$ suppressed hepatic cell death, and reduced levels of serum alanine aminotransferase and hepatic malondialdehyde. The results indicated the potential benefits of inhalation of hydro- 
gen gas as an applicable method to reduce oxidative stress. ${ }^{6}$

In Parkinson's disease, mitochondrial dysfunction and the associated oxidative stress are major causes of dopaminergic cell loss in the substantia nigra. Fu et al. examined effects of $\sim 50 \%$-saturated molecular hydrogen in drinking water before and after the stereotactic surgery on nigrostrital degeneration in a rat model of Parkinson's disease. Molecular hydrogen prevented both the development and progression of the nigrostrital degeneration. Preand post-treatment with hydrogen prevented the dopaminergic cell loss. The study suggested that hydrogen water was likely able to retard the development and progression of Parkinson's disease by reducing the oxidative stress. $^{7}$

Chronic physical restraint stress to mice enhanced levels of oxidative stress in the brain and impaired learning and memory. Consumption of hydrogen water ad- libitum throughout the whole period suppressed the increase in the oxidative stress and prevented cognitive impairment, whereas hydrogen water did not improve cognitive ability when no stress was provided. Thus, continuous consumption of hydrogen water reduces oxidative stress in the brain, and prevents the stressinduced decline in learning and memory caused by chronic physical restraint. The results showed that hydrogen water may be applicable for preventive use in cognitive or other neuronal disorders. ${ }^{8}$

Oxidative stress is recognized widely as being associated with diabetes. Hydrogen-rich water intake was associated with a trend of decreased serum concentrations of oxidized LDL and free fatty acids, and increased plasma levels of adiponectin and extracellular-superoxide dismutase. In 4 of 6 patients with impaired glucose tolerance (IGT), intake of hydrogen-rich water normalized the oral glucose tolerance test, these results suggest that supplementation with hydrogen-rich pure water had beneficial effects on lipid and glucose metabolism in humans. The results suggested that a sufficient supply of this water may prevent or delay development and progression of type- 2 diabetes mellitus and insulin resistance by providing protection against oxidative stress. ${ }^{9}$

\section{Reactive oxygen species play an important role in diabetes- associated erectile dysfunction}

ED is defined as the inability to achieve or maintain erections sufficient for satisfactory sexual intercourse. 18 Approximately 52\% of men between the age of 40 and 70 years suffer from ED due to various causes, such as pathophysiological changes in nerves, blood vesicles, corporal smooth muscle tissue, and endothelial cells. ${ }^{12,19}$ As most of these risk factors for development of ED are inherent to diabetes mellitus, the current estimates suggest that as many as $75 \%$ of men with diabetes will develop some degree of ED at an earlier age. ${ }^{20}$

Hyperglycemia induced overproduction of superoxide is an initiating event in the activation of pathways involved in the pathogenesis and complications from DM.21,22 0xidative stress-mediated neurovascular alteration appears to play an integral role in the development of ED in diabetic populations. ${ }^{10,11}$ Nitric oxide (NO) is the principal mediator of penile erection. ${ }^{12}$ Nitrergic nerves and endothelial cells directly release NO in the penis, where it stimulates guanylyl cyclase to produce cGMP and lowers intracellular calcium levels. ${ }^{23}$ This triggers the relaxation of arterial and trabecular smooth muscles leading to arterial dilatation, venous constriction, which result in a penile erection. ${ }^{24}$ The presence of oxygen free radicals inactivates NO and reduces its physiologic impact, $\mathrm{NO}$ is a free radical and can react with other radicals, such as superoxide anions, to produce peroxynitrite and contributes to numerous pathological conditions such as atherosclerosis and generalized reperfusion injury. ${ }^{13}$ Peroxynitrite is a highly toxic molecule which causes apoptosis, serious damage to cellular mechanisms, including small penile resistance arteries. ${ }^{21,22}$ High concentrations of peroxynitrite can produce highly ROS, which can initiate a cascade of redox reactions triggering apoptosis and evoke cytotoxic effects on endothelial and neuronal cells. Increased apoptosis and resulting disruption of cellular processes in turn lead to reduced expression levels of eNOS and nNOS. ${ }^{25}$ Additionally, increased auto-oxidation of glucose and oxidation of low-density lipoproteins have been described in diabetes, which may result in the overproduction of free radical species, leading to smooth muscle dysfunction. 26

It is postulated that the hydroxyl radical is generated in biological systems from superoxide anion and hydrogen peroxide by the HaberWeiss reaction or from hydrogen peroxide by the Fenton reaction. ${ }^{27,28}$ It is biologically important to eliminate hydroxyl radicals, because no enzyme detoxifies hydroxyl radicals. ${ }^{14,15}$ Hydroxyl radical is the strongest of the oxidant species and reacts indiscriminately with nucleic acids, lipids and proteins. ${ }^{3}$ Hydroxyl radicals play an important role in the diabetes-related ED. ${ }^{29,30}$

Previous studies indicate that diabetesinduced decreased NO levels in corporeal areas could be mainly due to the lack of essential cofactors for NOS activity. ${ }^{31}$ However, studies involving the systemic and cavernosal vasculature showed that superoxide-induced inactivation, rather than reduced activity of NOS, was the major cause of reduced NO levels. ${ }^{32}$

A growing number of studies suggest ROS play an important role in diabetic ED and antioxidants can markedly decrease the production of ROS and improve the erectile function. A study indicated that non-insulin dependent DM caused excessive generation of free radicals, decreased scavenging systems, marked elevation of malondialdehyde, and decreased glutathione levels in diabetic rats, all of which lead to ED. ${ }^{33}$ An in vitro study suggested that corpus cavernosal vascular smooth muscle cells (CCSMC) grown in a high glucose milieu exhibited changes in ROS which were significantly increased; after the cells were exposed to vitamin $\mathrm{E}$ for 14 days there was a reduction in ROS formation. ${ }^{34}$ ROS production was increased in penile tissue and erectile function was decreased in diabetic rats while the administration of the antioxidant $\alpha$-lipoic acid reduced the ROS production and improved the erectile function in diabetic ED rats. ${ }^{35}$ Khan et al. reported that overproduction of superoxide anion may play an important role in the development of diabetic ED. NO- and electrical field stimulated -mediated cavernosal smooth muscle relaxation was impaired in a rabbit model of diabetes but superoxide dismutase (SOD, the enzyme that accelerates the breakdown of the superoxide anion, $\mathrm{O}_{2}$-to $\mathrm{H}_{2} \mathrm{O}$ ) significantly reversed the impaired relaxation. ${ }^{36}$ Bivalacqua et al. reported that superoxide anion was increased in diabetic rats and that diabetic rats transfected with AdCMVECSOD had reduced corporal superoxide anion levels and increased cavernosal cGMP levels by increasing NO bioavailability. Adenoviral gene transfer of EC-SOD could restore erectile function by reducing the production of superoxide anion in diabetic rats. ${ }^{37}$ Kawakami et al. reported that the superoxide dismutase (SOD) mimetic tempol increased erectile function through modulating oxidative stress-related genes (SOD1, SOD2, GP x 1, CAT, NOS2, NOS3) in diabetic rats. ${ }^{38}$ of 84 reactive oxidative stress and antioxidant genes 32 were identified specific to diabetic rats compared to healthy controls. Tempol restored erectile function by reducing ROS and decreasing apoptosis in diabetic rats. ${ }^{39}$ Sildenafil treatment, the first-line pharmacotherapy for ED, rapidly and chronically improved vascular relaxation in diabetic rats by decreasing superoxide formation and membrane expression of the NAD(P)H oxidase subunit gp91phox and Rac. 40

\section{Hypothesis}

We hypothesize that hydrogen gas may be a promising, safe and effective agent for the treatment of diabetes-related ED by reducing levels of hydroxyl radicals and peroxynitrite.

Our hypothesis is based on the theory that molecular hydrogen can selectively reduce hydroxyl radical and peroxynitrite levels in vitro and in vivo, as hydroxyl radicals and per- 
oxynitrite are much more reactive than other ROS. $^{3}$

$\mathrm{H}_{2}$ has a number of advantages: i) it effectively neutralizes hydroxyl radicals in living cells, unlike most known antioxidants, which are unable to successfully target organelles; 3 ii) it has favorable distribution characteristics: it can penetrate biomembranes and diffuse into the cytosol, mitochondria and nucleus; 3 iii) despite the moderate reduction activity of $\mathrm{H}_{2}$, its rapid gaseous diffusion might make it highly effective for reducing cytotoxic radicals. Its ability to protect nuclear DNA and mitochondria suggests that it could reduce the risk of life style-related diseases and cancer: ${ }^{3}$ iv) it is likely that $\mathrm{H}_{2}$ is mild enough not to disturb metabolic oxidation reduction reactions or to disrupt ROS involved in cell signaling-unlike some antioxidant supplements with strong reductive reactivity, which increase mortality, possibly by affecting essential defensive mechanisms. ${ }^{3}$

We will design a study to prove our hypothesis. Diabetic ED rats are given $\mathrm{H}_{2}-$ saturated water for 8 weeks. After 8 weeks, we will measure the production of hydroxyl radicals, peroxynitrite, and NO, erectile function, liver and kidney function to observe if hydrogen administration improves erectile function without side effects through neutralizing hydroxyl radicals and peroxynitrite. We will also examine the production of the ROS involved in cell signaling to observe whether hydrogen disrupts their normal physiological function or not.

The safety of hydrogen for humans is demonstrated by its application in Hydreliox, an exotic breathing gas mixture of $49 \%$ hydrogen, $50 \%$ helium and $1 \%$ oxygen, which is used for the prevention of decompression sickness and nitrogen narcosis during very deep technical diving. ${ }^{41}$ Also no adverse effects have been found using drinking hydrogen water in a human study. ${ }^{42}$ A study showed that 20 subjects with potential metabolic syndrome consumed 1.5-2.0 $\mathrm{V} /$ day of $\mathrm{H}_{2}-$ saturated water for 8 weeks, the treated subjects showed significant improvements in liver and kidney function, ${ }^{43}$ which showed that $\mathrm{H}_{2}$ had no toxic side effects on liver and kidney and may even protect liver and kidney function. Another study also showed that hemodialysis patients treated with dialysis solution with $\mathrm{H}_{2}$ for 6 months had no adverse clinical signs or symptoms. 44

Hydrogen gas may be a promising, safe and effective agent for the treatment of diabetesrelated ED by reducing hydroxyl radical and peroxynitrite levels.

\section{References}

1. Buchholz BM, Kaczorowski DJ, Sugimoto $\mathrm{R}$, et al. Hydrogen inhalation ameliorates oxidative stress in transplantation induced intestinal graft injury. Am J Transplant 2008;8:2015-24.

2. Shen M, He J, Cai J, et al. Hydrogen as a novel and effective treatment of acute carbon monoxide poisoning. Med Hypotheses 2010;75:235-7.

3. Ohsawa I, Ishikawa M, Takahashi K, et al. Hydrogen acts as a therapeutic antioxidant by selectively reducing cytotoxic $0 x y-$ gen radicals. Nat Med 2007;13:688-94.

4. Hayashida K, Sano M, Ohsawa I, et al. Inhalation of hydrogen gas reduces infarct size in the rat model of myocardial ischemia-reperfusion injury. Biochem Biophys Res Commun 2008;373:30-5.

5. Sun Q, Kang Z, Cai J, et al. Hydrogen-rich saline protects myocardium against ischemia/reperfusion injury in rats. Exp Biol Med (Maywood) 2009;234:1212-9.

6. Fukuda $\mathrm{K}$, Asoh $\mathrm{S}$, Ishikawa $\mathrm{M}$, et al. Inhalation of hydrogen gas suppresses hepatic injury caused by ischemia/reperfusion through reducing oxidative stress. Biochem Biophys Res Commun 2007; 361:670-4.

7. Fu Y, Ito M, Fujita Y, et al. Molecular hydrogen is protective against 6-hydroxydopamine-induced nigrostriatal degeneration in a rat model of Parkinson's disease. Neurosci Lett 2009;453:81-5.

8. Nagata K, Nakashima-Kamimura N, Mikami T, Ohsawa I, Ohta S. et al. Consumption of molecular hydrogen prevents the stress-induced impairments in hippocampus-dependent learning tasks during chronic physical restraint in mice. Neuropsychopharmacology 2009;34:501-8.

9. Kajiyama S, Hasegawa G, Asano M, et al. Supplementation of hydrogen-rich water improves lipid and glucose metabolism in patients with type 2 diabetes or impaired glucose tolerance. Nutr Res 2008;28:13743.

10. Saenz de Tejada I, Goldstein I, Azadzoi K, et al. Impaired neurogenic and endothelium-mediated relaxation of penile smooth muscle from diabetic men with impotence. N Engl J Med 1989;320:1025-30.

11. Laight DW, Carrier MJ, Anggard EE. Antioxidants, diabetes and endothelial dysfunction. Cardiovasc Res 2000;47:45764.

12. Lue TF. Erectile dysfunction. N Engl J Med 2000;342:1802-13.

13. Beckman JS, Koppenol WH. Nitric oxide, superoxide, and peroxynitrite: the good, the bad, and ugly. Am J Physiol 1996;271:1424-1437.

14. Halliwell B, Gutteridge JM. Role of free radicals and catalytic metal ions in human disease: an overview. Methods Enzymol 1990;186:1-85.

15. Bektaşoğlu B, Esin Celik S, Ozyürek M, et al. Novel hydroxyl radical scavenging antioxidant activity assay for water-soluble antioxidants using a modified CUPRAC method. Biochem Biophys Res Commun 2006;345:1194-200.

16. Liu C, Cui J, Sun Q, Cai J. Hydrogen therapy may be an effective and specific novel treatment for acute radiation syndrome. Med Hypotheses 2010;74:145-6.

17. Schoenfeld MP, Ansari RR, Zakrajsek JF, et al. Hydrogen therapy may reduce the risks related to radiation-induced oxidative stress in space flight. Med Hypotheses 2011;76:117-8.

18. Kalsi JS, Kell PD. Update on oral treatments for male erectile dysfunction. J Eur Acad Dermatol Venereol 2004;18:267-74.

19. Burchardt T, Burchardt M, Karden J, et al. Reduction of endothelial and smooth muscle density in the corpora cavernosa of the streptozotocin induced diabetic rat. J Urol 2000;164:1807-11.

20. Yohannes E, Chang J, Tar MT, et al. Molecular targets for diabetes mellitusassociated erectile dysfunction. Mol Cell Proteomics 2010;9:565-78.

21. Jeremy JY, Jones RA, Koupparis AJ, et al. Reactive oxygen species and erectile dysfunction: possible role of NADPH oxidase. Int J Impot Res 2007;19:265-80.

22. Amaral S, Oliveira PJ, Ramalho-Santos J. Diabetes and the impairment of reproductive function: possible role of mitochondria and reactive oxygen species. Curr Diabetes Rev 2008;4:46-54.

23. Toda N, Ayajiki K, Okamura T. Nitric oxide and penile erectile function. Pharmacol Ther 2005;106:233-66.

24. Andersson KE, Stief CG. Neurotrans mission and the contraction and relaxation of penile erectile tissues. World $\mathrm{J}$ Urol 1997;15:14-20.

25. Evgenov OV, Liaudet L. Role of nitrosative stress and activation of poly (ADP-ribose) polymerase-1 in cardiovascular failure associated with septic and hemorrhagic shock. Curr Vasc Pharmacol 2005;3:293-9.

26. Baynes JW, Thorpe SR. Role of oxidative stress in diabetic complications: a new perspective on an old paradigm. Diabetes 1999;48:1-9.

27. Halliwell B, Gutteridge JM. Biologically relevant metal ion-dependent hydroxyl radical generation. An update. FEBS Lett 1992;307:108-12.

28. Halliwell B, Gutteridge JM. Oxygen free radicals and iron in relation to biology and medicine: some problems and concepts. Arch Biochem Biophys 1986;246:501-14.

29. Zamblé A, Yao D, Martin-Nizard F, et al. Vasoactivity and antioxidant properties of Microdesmis keayana roots. J Ethnopharmacol 2006;104:263-9.

30. Evgenov OV, Liaudet L. Role of nitrosative 
stress and activation of poly(ADP-ribose) polymerase-1 in cardiovascular failure associated with septic and hemorrhagic shock. Curr Vasc Pharmacol 2005;3:293-9.

31. Gur S, Ozturk B, Karahan ST. Impaired endothelium-dependent and neurogenic relaxation of corpus cavernosum from diabetic rats: improvement with L-arginine. Urol Res 2000;28:14-9.

32. Laight DW, Carrier MJ, Anggard EE. Antioxidants, diabetes and endothelial dysfunction. Cardiovasc Res 2000;47:45764.

33. Ryu JK, Kim DJ, Lee T, et al. The role of free radical in the pathogenesis of impotence in streptozotocin-induced diabetic rats. Yonsei Med J 2003;44:236-41.

34. Ganz MB, Seftel A. Glucose-induced changes in protein kinase $\mathrm{C}$ and nitric oxide are prevented by vitamin E. Am J Physiol Endocrinol Metab 2000;278:E14652.

35. Keegan A, Cotter MA, Cameron NE. Effects of diabetes and treatment with the antioxidant alpha-lipoic acid on endothelial and neurogenic responses of corpus cavernosum in rats. Diabetologia 1999;42:343-
50.

36. Khan MA, Thompson CS, Jeremy JY, et al. The effect of superoxide dismutase on nitric oxide-mediated and electrical fieldstimulated diabetic rabbit cavernosal smooth muscle relaxation. BJU Int 2001;87:98-103.

37. Bivalacqua TJ, Usta MF, Kendirci M, et al. Superoxide anion production in the rat penis impairs erectile function in diabetes: influence of in vivo extracellular superoxide dismutase gene therapy. J Sex Med 2005;2:187-98.

38. Kawakami T, Urakami S, Hirata $\mathrm{H}$, et al. Superoxide dismutase analog (Tempol: 4hydroxy-2, 2, 6, 6-tetramethylpiperidine 1oxyl) treatment restores erectile function in diabetes-induced impotence. Int J Impot Res 2009;21:348-55.

39. Hirata H, Kawamoto K, Kikuno N, et al. Restoring erectile function by antioxidant therapy in diabetic rats. J Urol 2009;182:2518-25.

40. Schäfer A, Fraccarollo D, Pförtsch S, et al. Improvement of vascular function by acute and chronic treatment with the PDE-5 inhibitor sildenafil in experimental dia- betes mellitus. Br J Pharmacol 2008;153: 886-93.

41. Abraini JH, Gardette-Chauffour MC, Martinez E, et al. Psychophysiological reactions in humans during an open sea dive to $500 \mathrm{~m}$ with a hydrogen-heliumoxygen mixture. J Appl Physiol 1994;76: 1113-8.

42. Kajiyama S, Hasegawa G, Asano M, et al. Supplementation of hydrogen-rich water improves lipid and glucose metabolism in patients with type 2 diabetes or impaired glucose tolerance. Nutr Res 2008;28:13743.

43. Nakao A, Toyoda Y, Sharma P, et al. Effectiveness of hydrogen rich water on antioxidant status of subjects with potential metabolic syndrome-an open label pilot study. J Clin Biochem Nutr 2010;46: 140-9.

44. Nakayama M, Nakano H, Hamada H, et al. A novel bioactive haemodialysis system using dissolved dihydrogen (H2) produced by water electrolysis: a clinical trial. Nephrol Dial Transplant 2010;25:3026-33. 